\title{
Apport de la modélisation numérique dans l'analyse des risques sismiques liés à la liquéfaction
}

\section{F. LOPEZ-CABALLERO}

A. MODARESSI-

FARAHMAND-RAZAVI

Laboratoire MSSMat CNRS

UMR 8579

École centrale Paris

Grande Voie des Vignes

92290 Châtenay-Malabry

L'objectif de ce travail est d'étudier les effets du comportement non linéaire du sol, introduit par la liquéfaction, sur l'interaction sol-structure non linéaire lors d'un séisme en utilisant des simulations numériques. On évalue aussi l'efficacité d'une méthode de renforcement de sols dans la réduction du potentiel de liquéfaction d'un profil de sol. Les effets favorables ou défavorables de l'utilisation d'inclusions rigides dans la réponse sismique d'une structure reposant sur un profil de sol liquéfiable sont présentés. Cette analyse montre l'efficacité de la méthode à réduire la liquéfaction et par conséquent les tassements induits mais elle montre aussi que cette intervention modifie les caractéristiques dynamiques du signal à la surface et en conséquence augmente le niveau des déplacements subits par la structure.

Mots-clés : liquéfaction, modèle constitutif, méthode d'amélioration du sol, interaction-sol structure.

\section{Contribution of numerical modeling in the liquefaction seismic risks assessment}

The present paper deals with the influence of soil non-linearity, introduced by soil liquefaction, on the soil-foundationstructure interaction phenomena. Numerical simulations are carried out so as to study an improvement method to reduce the liquefaction potential in a sandy soil profile subjected to a shaking. The efficiency of the confinement walls in the mitigation of a liquefiable soil is showed. However, the intervention at the foundation soil modifies the dynamic characteristics of soil-structure system and it seems to be an unfavorable method from the structural point of view.

Key words: liquefaction, constitutive model, mitigation method, soil structure interaction. 


\section{Introduction}

Dans le domaine de la gestion des risques naturels, il est communément admis que le risque est le produit de convolution entre l'aléa et la vulnérabilité des éléments exposés. Pour atténuer ces risques, il faut savoir quantifier et si possible réduire chacune de ses composantes.

La démarche courante de l'évaluation du risque sismique est constituée de quatre étapes :

- la définition du mouvement sismique : ce mouvement est déterminé en fonction des caractéristiques de la source sismique, des propriétés du trajet source-site et des effets de site. Les effets de sites englobent la topographie et les effets locaux dus au comportement des sols. Il faut également y inclure les effets induits tels que les mouvements de terrain et la liquéfaction des sols ;

- l'estimation de l'endommagement des ouvrages soumis au mouvement sismique : celle-ci peut être affectée selon les cas par l'histoire du chargement (effet réplique) et par des effets relatifs à l'interaction entre ces ouvrages et le sol encaissant ;

- l'évaluation de l'impact de ces dommages sur le fonctionnement des ouvrages considérés ;

- l'estimation des impacts socio-économiques liés à l'endommagement physique des ouvrages et la perte de leur fonctionnement.

Ici, nous nous intéressons au phénomène de liquéfaction et nous nous focalisons plus spécifiquement sur les effets locaux et l'interaction sol-structure.

Le phénomène de liquéfaction des sables lors de séismes constitue un domaine de préoccupation important dans la géotechnique et le génie parasismique. Lévaluation du risque de liquéfaction dans un site donné, de ses conséquences éventuelles et des possibles solutions à ce problème constitue un enjeu économique et social important. Ce risque peut être exprimé par l'intermédiaire d'un coefficient de sécurité à la liquéfaction, qui prend en compte la résistance du sol par rapport aux efforts produits par le séisme. Dans la pratique, du fait des difficultés rencontrées pour modéliser analytiquement les conditions du sol sableux in situ, l'évaluation du risque de liquéfaction se réalise à l'aide des méthodes empiriques ou simplifiées (Seed et Idriss, 1971 ; Martin et Seed, 1979 ; Seed et al., 1983 ; Youd et al., 2001).

Les caractéristiques et les propriétés des matériaux sont définies à l'aide des essais au laboratoire ou in situ. Cependant, les contraintes et les déformations induites par le séisme dans le profil du sol sont estimées à partir de calculs avec des modèles en contraintes totales. Ces types de modèles ne sont pas adaptés pour estimer le comportement du sable (i.e. comportement contractant ou dilatant) et par conséquent l'évolution de la pression interstitielle lors du chargement sismique.

En vue de remédier aux problèmes de liquéfaction dans les sols, les différents guides de construction parasismique (e.g. NRC, 1985) proposent des solutions telles que la baisse de la nappe phréatique et le compactage des couches où le sol est amélioré dans sa masse ou l'utilisation des inclusions pour le renforcer. Dans ce travail, nous testons la performance de ce dernier type de solution. Pour cela, nous utilisons le logiciel GEFDyn (Aubry et al., 1986 ; Aubry et Modaressi,
1996) permettant une modélisation du sol en termes de contraintes effectives par la méthode des éléments finis. Afin de prendre en compte le comportement du sable dans la réponse du profil, le sol est modélisé à l'aide d'un modèle de comportement élastoplastique (Hujeux, 1985). Ce modèle permet de simuler entre autres des phénomènes tels que la mobilité cyclique et la densification du sable lors de chargements. Les effets induits par la liquéfaction sur le mouvement du sol sur la réponse dynamique d'une structure sont étudiés pour analyser l'efficacité d'utilisation des inclusions rigides contre ce risque.

\section{2 \\ Modèle numérique}

Le modèle éléments finis est composé de trois parties : le sol de fondation, une fondation superficielle rigide et une structure représentée par un oscillateur à un seul degré de liberté. La géométrie et le maillage du modèle numérique proposé sont donnés sur la figure 1a. La structure correspond à un bâtiment d'une hauteur totale de $6 \mathrm{~m}$ avec une fondation de $6 \mathrm{~m}$ de longueur. Sa période fondamentale sur base fixe $\left(\mathrm{T}_{\mathrm{str}}\right)$ est de $0,4 \mathrm{~s}$. En ce qui concerne le sol de fondation, un modèle typique de sol stratifié/rocher déformable est considéré. Le profil de sol se compose principalement de couches d'argile recouvertes de $22 \mathrm{~m}$ du sable lâche (i.e. une densité relative $\mathrm{Dr}<50 \%$ ). L'épaisseur totale du profil de sol est de $40 \mathrm{~m}$ sur le rocher. Le modèle numérique est construit à partir des mesures in situ de SPT-N ${ }_{60}$ et de vitesse de propagation des ondes de cisaillement (Vs) données sur la figure $1 \mathrm{~b}$ (LopezCaballero et Modaressi-Farahmand-Razavi, 2008). La période fondamentale élastique du profil de sol est de 0,57 s. D’après les résultats du SPT et la description du sol, on déduit que la liquéfaction peut apparaître au niveau des couches entre les profondeurs de $4 \mathrm{~m}$ et $15 \mathrm{~m}\left(\mathrm{SPT}_{-\mathrm{N}}\right.$ entre 4 et 10). Comme à partir de $29 \mathrm{~m}$ de profondeur le profil de sol est composé principalement des argiles surconsolidées, le modèle élastoplastique est utilisé uniquement pour simuler le comportement des sols des premiers $29 \mathrm{~m}$. Dans ces couches, le module de cisaillement du sol augmente avec la profondeur. Pour les couches entre $29 \mathrm{~m}$ et 40 $\mathrm{m}$, un comportement élastique linéaire isotrope est supposé. Le rocher déformable est placé à $40 \mathrm{~m}$ de profondeur.

Des simulations 2D couplées (formulation $u-p_{w}$ ) en déformation plane par éléments finis ont été réalisées à l'aide du logiciel GEFDyn (Aubry et al., 1986 ; Aubry et Modaressi, 1996). Afin d'obtenir une meilleure estimation de la distribution de contraintes in situ et l'équilibre des efforts entre la structure et le sol en déformation plane, une modification concernant l'épaisseur de la couche du sol, ( déformation plane avec épaisseur ») (Saez, 2009), a été supposée. Dans ce cas, une épaisseur de $4 \mathrm{~m}$ est utilisée. Le sol saturé est simulé par des éléments quadratiques isoparamétriques avec huit nouds pour décrire les déplacements du solide et la pression du fluide. L'épaisseur de chaque élément est de 0,5 m. Un schéma d'intégration numérique implicite de Newmark avec $\gamma=0,625$ et $\beta=0,375$ est utilisé lors des analyses dynamiques (Katona et Zienkiewicz, 1985). 


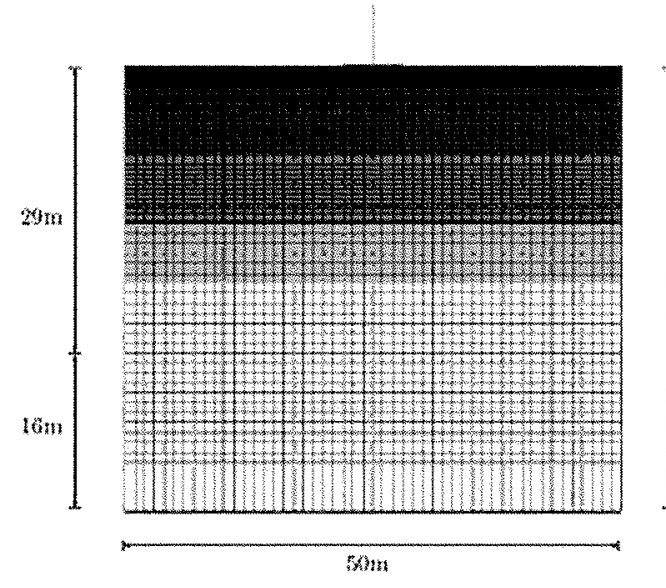

(a)
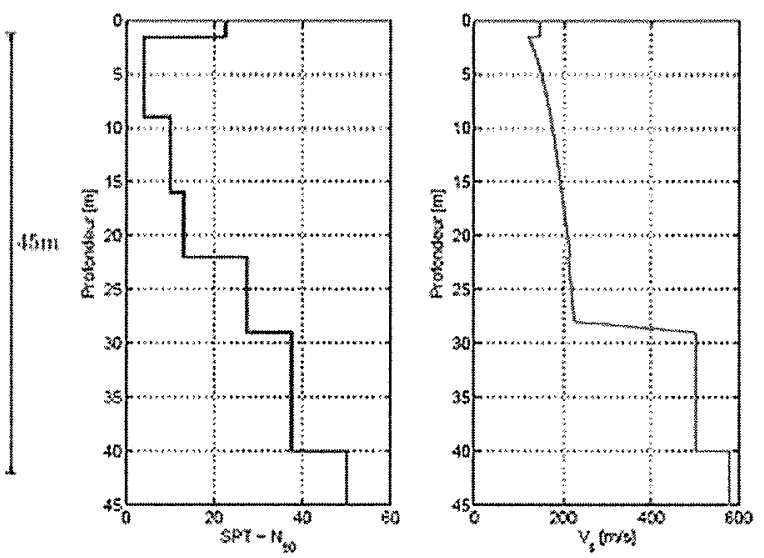

(b)

nc. 1 a) Maillage d'éléments finis pour le modèle numérique et b) profils de SPT et de vitesse de propagation des ondes $S$ pour le site d'étude et utilisés dans l'analyse numérique.

\section{9xis}

\section{Conditions aux limites}

Dans les analyses, seulement les ondes incidentes verticales sont prises en compte et comme l'on simule la réponse d'un semi-espace infini, des frontières équivalentes sont imposées aux nœuds latéraux (i.e. les déplacements des nœuds à la même profondeur des frontières opposées sont égaux dans toutes les directions). Concernant les conditions aux limites au niveau du rocher, des éléments paraxiaux représentant un « demi-espace élastique infini » ont été utilisés (Modaressi et Benzenati, 1994). Les ondes incidentes définies au rocher affleurant sont introduites à la base du modèle après déconvolution. Ainsi, le mouvement obtenu au rocher est la somme des ondes incidentes et des ondes réfléchies. Le rocher est supposé imperméable et la nappe phréatique est placée à $1 \mathrm{~m}$ de profondeur.

\section{5:}

\section{Modèle de sol}

Le modèle élastoplastique multimécanisme développé à l'École centrale Paris, connu comme modèle ECP (Aubry et al., 1982 ; Hujeux, 1985) est utilisé pour représenter le comportement du sol. Ce modèle permet de simuler le comportement du sol dans une large gamme de déformations. Il est écrit en termes des contraintes effectives. Tous les phénomènes irréversibles sont représentés par le couplage de quatre mécanismes plastiques élémentaires : trois mécanismes plastiques de déformations déviatoires et un mécanisme de déformation isotrope ou volumique. Les trois mécanismes déviatoires correspondent à trois mécanismes en déformations planes dans trois plans orthogonaux. Il utilise un critère de rupture de type Coulomb et intègre le concept d'état critique. L'évolution de l'écrouissage dépend de la déformation plastique (déviatoire et volumique pour les mécanismes déviatoires et volumique pour le mécanisme isotrope). Pour prendre en compte le comportement cyclique, un écrouissage cinématique fonction des variables d'état au dernier changement de charge est utilisé. Le comportement du sol est décomposé en trois domaines : pseudo-élastique, hystérétique et mobilisé. Pour plus de détailles sur le modèle ECP voir Hujeux (1985), Lopez-Caballero et Modaressi-Farahmand-Razavi (2008) et Modaressi-Farahmand-Razavi (2010) entre autres.

\section{Modèle de comportement pour la structure}

Afin de modéliser le comportement non linéaire de la structure, on utilise des éléments poutre continus non linéaires. Le comportement non linéaire est pris en compte par l'intermédiaire d'un modèle élastique parfaitement plastique dans le plan contraintedéformation normales $\left(\sigma_{n}-\varepsilon_{n}\right)$ (Fig. 2a). Le diagramme moment-courbure obtenu avec cette approche est donné sur la figure $2 b$. Les caractéristiques du modèle à un degré de liberté pour la structure utilisée dans cette étude sont : module d'élasticité, $E=25,5 \mathrm{GPa}$; contrainte seuil, $\sigma_{v}=6,0 \mathrm{MPa}$; masse, $\mathrm{M}=20000 \mathrm{~kg}$ et hauteur, $h=6 m$ (Saez, 2009). Avec ces caractéristiques la période fondamentale sur base fixe $\left(\mathrm{T}_{\mathrm{str}}\right)$ est de $0,4 \mathrm{~s}$.
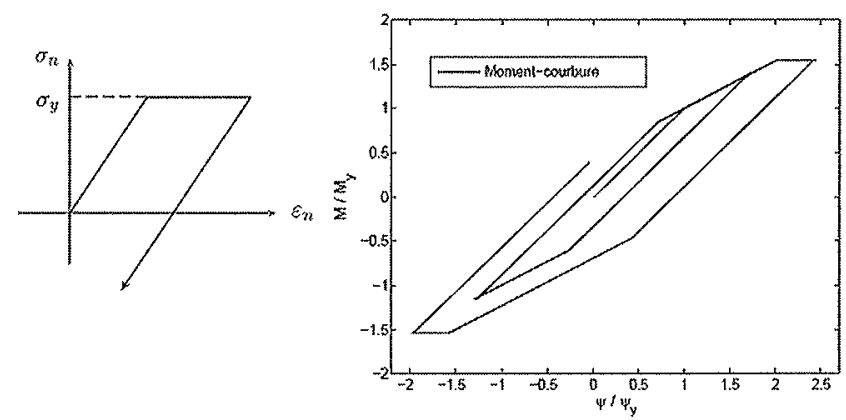

(a)

(b)

Fic. 2 Caractéristiques du modèle à un degré de liberté pour la structure (Saez, 2009). a) Loi de comportement $\left(\sigma_{n}-\varepsilon_{n}\right)$ et b) courbe moment-courbure. 
La courbe de capacité ou spectre de capacité de la structure obtenue par la méthode push-over ou poussée progressive est donnée sur la figure 3.

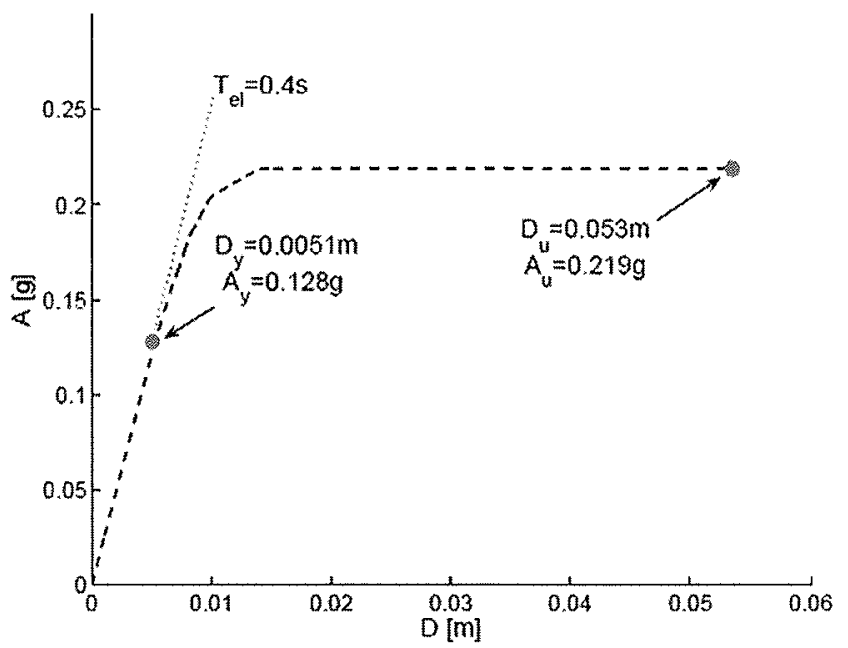

FG. 3 Spectre de capacité de la structure à partir de la méthode push-over.

\section{-}

\section{Signaux sismiques d'entrée}

Afin de définir les signaux sismiques d'entrée appropriés pour l'analyse dynamique couplée non linéaire, des signaux sismiques synthétiques ont été générés par un processus stochastique non stationnaire. Le modèle est adapté de celui proposé par Pousse et al. (2006). La méthode repose sur quatre indicateurs communs en génie parasismique : l'accélération maximale, la durée du mouvement fort, l'intensité d'Arias (Arias, 1970) et la fréquence centrale. Ces indicateurs sont empiriquement reliés à une base de données par l'intermédiaire des équations de prédiction des mouvements sismiques. Les événements s'étendent dans une gamme de magnitudes entre 6,5 et 7,0 , ils ont une distance du site à source de $50 \mathrm{~km}$ et des conditions de sol dense (i.e. $360 \mathrm{~m} / \mathrm{s}<\mathrm{V}_{\text {s } 30 \mathrm{~m}}<800 \mathrm{~m} / \mathrm{s}$ ).
Concernant le spectre de réponse des signaux d'entrée, le spectre médian, moyen \pm un écart-type et les courbes des spectres de réponse avec une probabilité de dépassement (PE) entre 2,75 et 97,5\% sont donnés sur la figure 4 . On peut remarquer que le spectre de réponse médian est compatible avec le spectre de réponse du sol type $\mathrm{A}$ de l'Eurocode8 ajusté à la valeur médiane de l'accélération maximale en rocher affleurant. Les statistiques sur quelques caractéristiques des signaux d'entrée sont résumées dans le tableau I. Ces caractéristiques sismiques sont l'accélération maximale au rocher affleurant (PHA), l'intensité d'Arias $\left(\mathrm{I}_{\text {Arias }}\right)$, la période prédominante (l'p), la période moyenne ('l'm) (Rathje et al., 1998), l'intensité spectrale (SI), la vitesse maximale (pgv), l'intensité rms $\left(\mathrm{I}_{\mathrm{rms}}\right.$ ) (Koutsourelakis et al., 2002), la densité d'énergie spécifique (SED) et la durée significative $\left(t_{595}\right)$. Pour les définitions des paramètres voir l'annexe $A$.

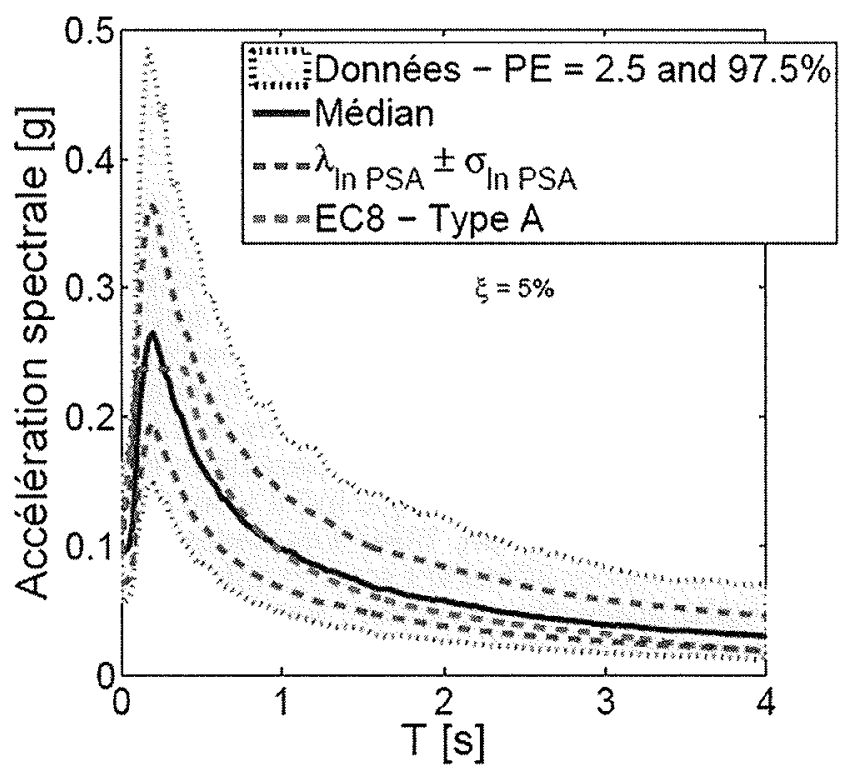

FG. 4 Spectre de réponse pour les signaux d'entrée.

TABLEAI Caractéristiques statistiques des séismes d'entrée.

\begin{tabular}{|c|c|c|c|c|c|}
\hline Parametre & Plage & Moyenre & (v) $[\%)$ & Nedarne & o. \\
\hline PHA $[g]$ & $0,05-0,19$ & 0,10 & 30 & 0,09 & 0,30 \\
\hline $\operatorname{Tm}[\mathrm{s}]$ & $0,36-0,72$ & 0,50 & 12 & 0,49 & 0,12 \\
\hline $\mathrm{Tp}[\mathrm{s}]$ & $0,10-0,50$ & 0,22 & 30 & 0,20 & 0,28 \\
\hline $\mathrm{I}_{\text {Arias }}[\mathrm{m} / \mathrm{s}]$ & $0,05-0,45$ & 0,20 & 54 & 0,17 & 0,55 \\
\hline$t_{595}[\mathrm{~s}]$ & $9,86-26,45$ & 20,40 & 16 & 20,65 & 0,17 \\
\hline Irms $\left[\mathrm{m} / \mathrm{s}^{2}\right]$ & $0,07-0,26$ & 0,14 & 29 & 0,13 & 0,29 \\
\hline$N_{\text {cyceq }}[]$. & $11-32$ & 21 & 19 & 21 & - \\
\hline $\mathrm{PGV}[\mathrm{cm} / \mathrm{s}]$ & $5,54-35,03$ & 15,06 & 37 & 0,14 & 0,37 \\
\hline $\mathrm{SI}[\mathrm{m}]$ & $0,18-0,76$ & 0,39 & 31 & 0,37 & 0,31 \\
\hline $\mathrm{SED}\left[\mathrm{cm}^{2} / \mathrm{s}\right]$ & $77,14-3319$ & 616 & 73 & 484 & 0,70 \\
\hline
\end{tabular}




\section{Simulation des essais de laboratoire}

Les paramètres du modèle ECP pour chaque couche ont été déterminés avec la méthodologie expliquée dans Santos et al. (2003) et Lopez-Caballero et al. (2007). Ils sont résumés dans le tableau II. Afin de vérifier les paramètres du modèle ainsi que de caractériser la résistance à la liquéfaction du sable placé entre 2 et $9 \mathrm{~m}$ de profondeur, son comportement a été étudié en simulant des essais de cisaillement cycliques drainés et non drainés.

Les résultats des simulations des essais de cisaillement cyclique à déformation imposée (i.e. $\mathrm{G} / \mathrm{G}_{\max }-\gamma$ et $\mathrm{D}-\gamma$ ) pour 3 niveaux de confinement (i.e. 20,50 et $70 \mathrm{kPa}$ ) sont montrés dans la figure 5 . Les courbes issues des simulations sont comparées à celles données par Seed et al. (1986). On remarque que dans ce cas en particulier, il existe une bonne correspondance entre les courbes calculées et celles mesurées expérimentalement. Pour des déformations supérieures à 0,01\% l'amortissement D est surestimé.

Les courbes obtenues pour la résistance au cisaillement cyclique (RCC) en fonction du nombre de cycles de charge pour produire la liquéfaction $(\mathrm{N})$ pour 2 niveaux de confinement (i.e. $\sigma_{m}^{\prime}=40$ et $50 \mathrm{kPa}$ ) sont donnés sur la figure $6 \mathrm{~b}$. Les résultats des tests simulés sont comparés avec les courbes de référence données par Seed et Idriss (1982) pour des sables à des densités différentes (i.e. valeurs de SPT). On remarque que les courbes obtenues correspondent relativement bien à celle correspondant à SPT- $\mathrm{N}_{60}=5$.

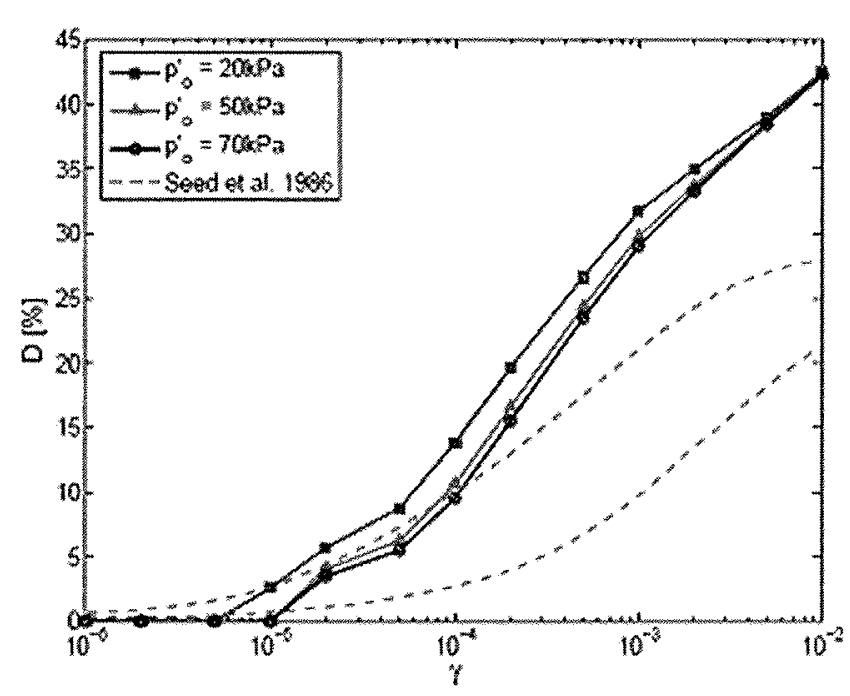

(b)

(a)

HG. 5 Comparaison entre la simulation et les courbes de références obtenues par Seed et al. (1986). a) $G / G_{\max }{ }^{-\gamma}$ et b) D- $\%$.

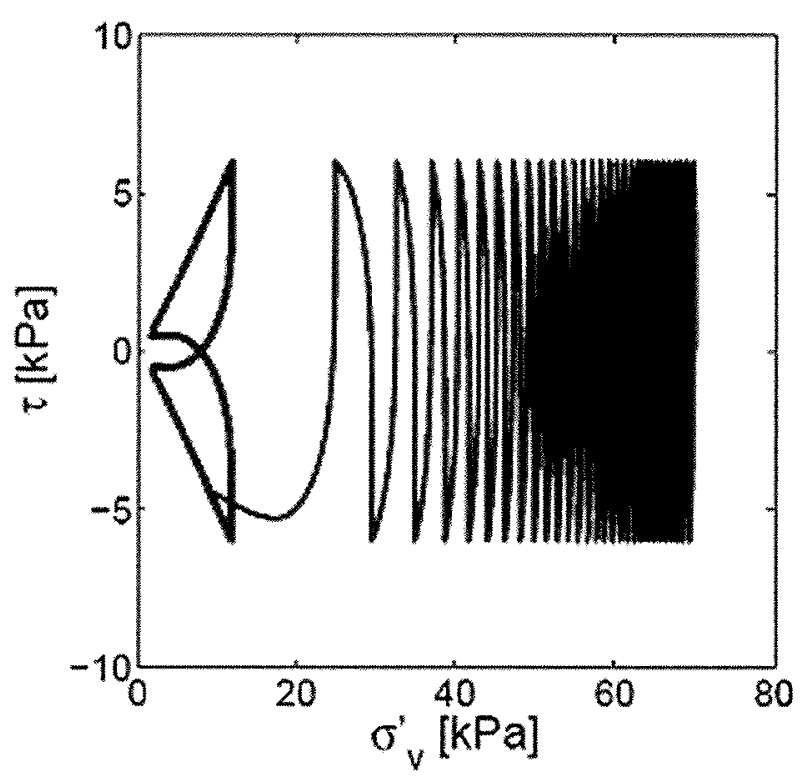

(a)

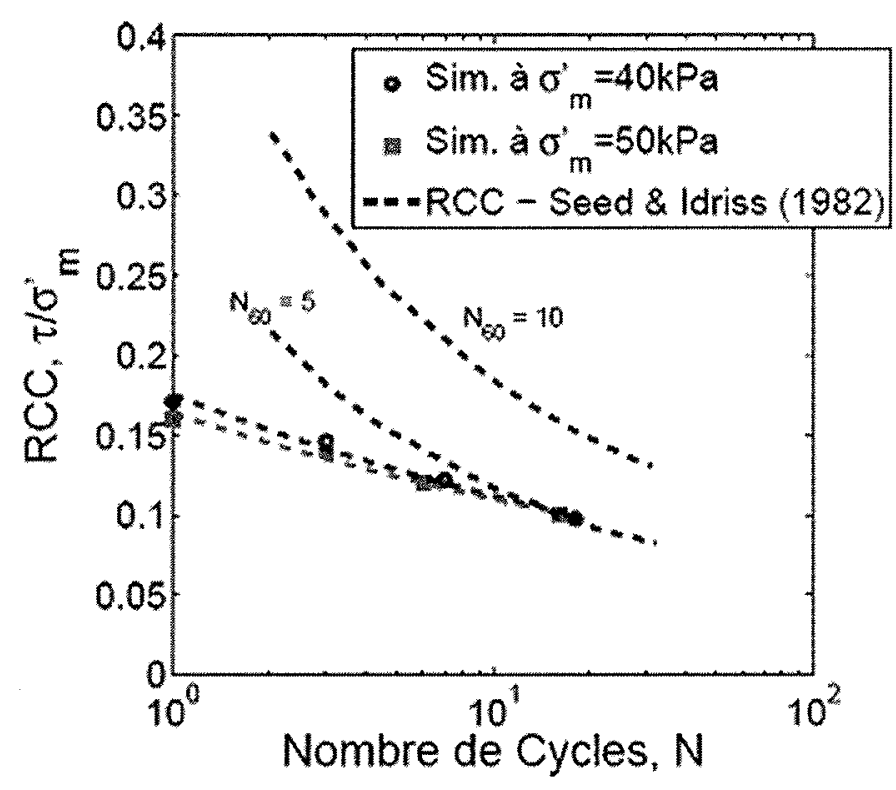

(b)

FG. 6 a) Réponse obtenue lors de la simulation d'un essai cyclique non drainé et b) courbes obtenues pour la résistance au cisaillement cyclique (RCC). 
TABLequ II Paramètres du modèle ECP pour les différentes couches du profil de sol.

\begin{tabular}{|c|c|c|c|c|c|c|}
\hline $\mathrm{ks}\lceil\mathrm{m} / \mathrm{s}\rfloor$ & $\begin{array}{c}0-1,5 \mathrm{~m} \\
110^{-2}\end{array}$ & $\begin{array}{c}1,5-2 \mathrm{~m} \\
110^{-5}\end{array}$ & $\begin{array}{l}2-9 \mathrm{~m} \\
110^{-5}\end{array}$ & $\begin{array}{c}9-16 \mathrm{~m} \\
110^{-5}\end{array}$ & $\begin{array}{c}16-22 \mathrm{~m} \\
110^{-4}\end{array}$ & $\begin{array}{c}22-29 \mathrm{~m} \\
110^{-4}\end{array}$ \\
\hline \multicolumn{7}{|c|}{ titisticite } \\
\hline $\mathrm{K}_{\mathrm{ref}}[\mathrm{MPa}]$ & 90 & 628 & 628 & 628 & 628 & 444 \\
\hline$G_{\text {ref }}[\mathrm{MPa}]$ & 45 & 290 & 290 & 290 & 290 & 222 \\
\hline $\mathrm{n}_{\mathrm{e}}$ & 0,0 & 0,5 & 0,5 & 0,5 & 0,5 & 0,4 \\
\hline \multicolumn{7}{|c|}{ Etat critique et plasticité } \\
\hline$\phi_{p p}^{\prime}\left[{ }^{\circ}\right]$ & 31 & 30 & 30 & 30 & 30 & 31 \\
\hline$\beta$ & 20 & 33 & 33 & 33 & 33 & 43 \\
\hline$d$ & 2,0 & 2,0 & 2,0 & 2,0 & 2,0 & 3,5 \\
\hline $\mathrm{b}$ & 0,2 & 0,2 & 0,2 & 0,2 & 0,2 & 0,2 \\
\hline $\mathrm{p}_{c o}^{\prime}[\mathrm{MPa}]$ & 1,86 & 0,02 & 0,04 & 0,05 & 0,08 & 1,80 \\
\hline \multicolumn{7}{|c|}{ Écrouissage deviatoire et Isotrope } \\
\hline$\psi\left[{ }^{\circ}\right]$ & 31 & 30 & 30 & 30 & 30 & 31 \\
\hline a1 & 0,0001 & 0,0001 & 0,0001 & 0,0001 & 0,0001 & 0,0001 \\
\hline $\mathrm{a} 2$ & 0,0002 & 0,002 & 0,005 & 0,0050 & 0,0050 & 0,0040 \\
\hline c & 0,0010 & 0,002 & 0,004 & 0,004 & 0,004 & 0,0600 \\
\hline $\mathrm{m}$ & 1,0 & 1,5 & 1,5 & 1,5 & 1,5 & 1,0 \\
\hline \multicolumn{7}{|c|}{ Domanes de connporternent } \\
\hline rela & 0,013 & 0,03 & 0,03 & 0,03 & 0,03 & 0,005 \\
\hline rhys & 0,220 & 0,04 & 0,04 & 0,04 & 0,04 & 0,03 \\
\hline$r^{m o b}$ & 0,800 & 0,80 & 0,80 & 0,80 & 0,80 & 0,80 \\
\hline \multicolumn{7}{|c|}{ Pour tous les cas $p_{\mathrm{cc}}=1,0 \mathrm{MPa}$ et $a_{4}=1$} \\
\hline
\end{tabular}

\section{3}

\section{Analyse de la liquéfaction}

\section{1}

\section{Réponse en champ libre}

Afin de définir à la fois les profondeurs, et le niveau d'accélération pour lesquels la liquéfaction a lieu, les réponses en champ libre du profil de sol sont analysées. Lincrément de pression interstitielle $\left(\Delta p_{w}\right)$ dans le profil de sol obtenu à la fin du signal sismique (i.e. analyse co-sismique) pour toutes les simulations ainsi que la contrainte effective verticale initiale $\sigma^{\prime}$ sont donnés sur la figure 7 . On observe qu'en ce qui concerne la réponse moyenne obtenue, la liquéfaction n'apparaît pas (i.e. $\Delta \mathrm{p}_{\mathrm{w}}<\sigma_{\mathrm{vo}}^{\prime}$ ). Sinon, concernant toutes les simulations, dans certains cas, l'apparition de la liquéfaction se trouve au niveau des couches entre 2 et $15 \mathrm{~m}$ de profondeur. En supposant que la liquéfaction apparait lorsque le rapport de surpression interstitielle $\left(\mathrm{ru}=\Delta \mathrm{p}_{\mathrm{w}} / \sigma_{\mathrm{vo}}^{\prime}\right)$ est supérieur à 0,8 , un profil de probabilité de liquéfaction peut être estimé. La probabilité de liquéfaction est estimée comme $p_{f}(z)=N_{f}(z) / N$, où $N_{f}(z)$ est le nombre de simulations où $r u \geq 0,8$ à la profondeur $z$ et $N$ est le nombre total de simulations. En utilisant cette approche, un profil de prob[ru $\geq 0,8]$ en fonction de la profondeur est donné sur la figure 8. Selon ces résultats, la probabilité de liquéfaction maximale est de $12 \%$ entre 4 et $6 \mathrm{~m}$ de profondeur.

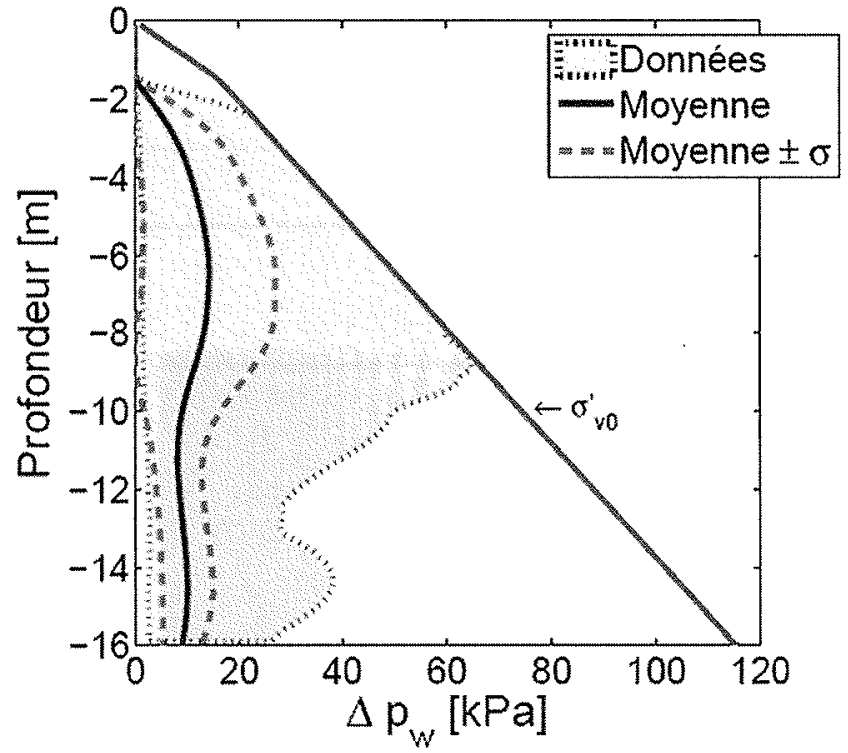

FIG. 7 Incrément de la pression interstitielle obtenu dans le profül de sol (champ libre).

Des travaux récents concernant l'évaluation des dommages causés par la liquéfaction sur les constructions (Juang et al., 2005 ; Bird et al., 2006), soulignent la nécessité de définir l'épaisseur de la couche liquéfié afin d'estimer la déformation permanente du sol. En vue de quantifier l'extension de la zone liquéfiée, on 


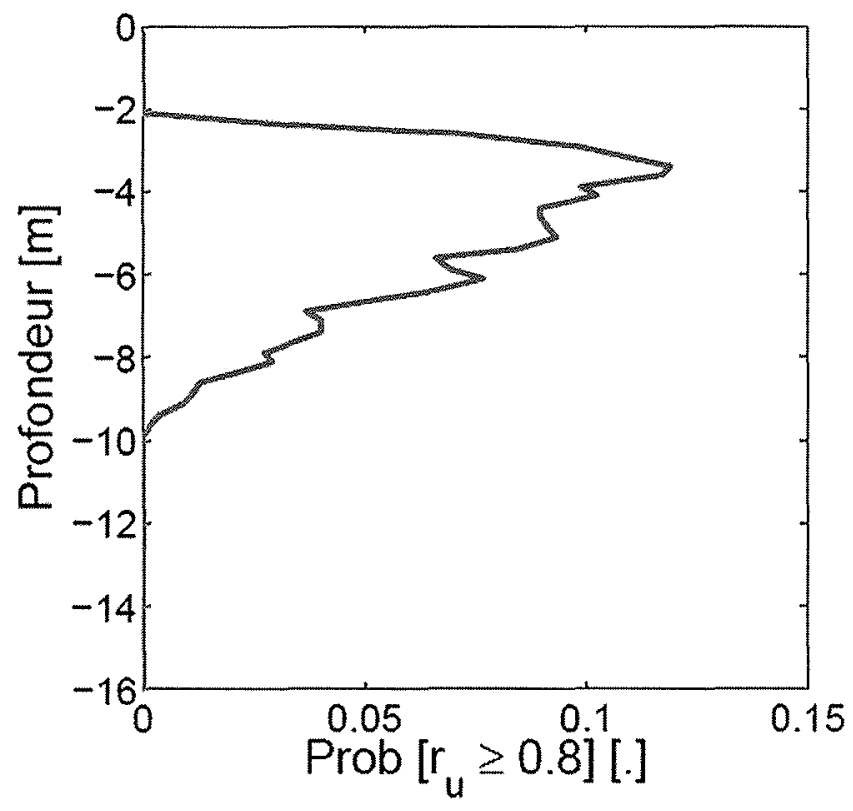

HC. 8 Évolution de la probabilité de liquéfaction avec la profondeur (champ libre).

utilise l'indice de liquéfaction $(Q)$ calculé pour le profil. Ce paramètre est défini par Shinozuka et Ohtomo (1989) comme :

$$
Q(t)=\frac{1}{H} \int_{0}^{H} \frac{\Delta p_{w}(t, z)}{\sigma_{v o}^{\prime}(z)} d z
$$

où $\mathrm{H}$ est la profondeur choisie (dans ce cas, $\mathrm{H}=$ $10 \mathrm{~m}), \Delta \mathrm{p}_{\mathrm{w}}(\mathrm{t}, \mathrm{z})$ est l'incrément de pression interstitielle au temps $t$ et à la profondeur $z$. Afin de représenter l'évolution de $\mathrm{Q}_{\mathrm{H}}=10 \mathrm{~m}$ en fonction d'un paramètre de sévérité sismique (e.g. paramètres du tableau I), on réalise une analyse en composantes principales des réponses obtenues (Fig. 9a). Il est constaté que les variables qui représentent le mieux l'évolution de $\mathrm{Q}_{\mathrm{H}}=10 \mathrm{~m}$ sont PHA, $\mathrm{I}_{\text {Arjas }}$ et $\mathrm{I}_{\mathrm{rms}}$. En vue d'évaluer la sensibilité de $\mathrm{Q}_{\mathrm{H}}=10 \mathrm{~m}$ a ces trois variables en éliminant l'effet des corrélations entre elles, l'indice de corrélation partielle (PCC) a été utilisé (Fig. 9b).

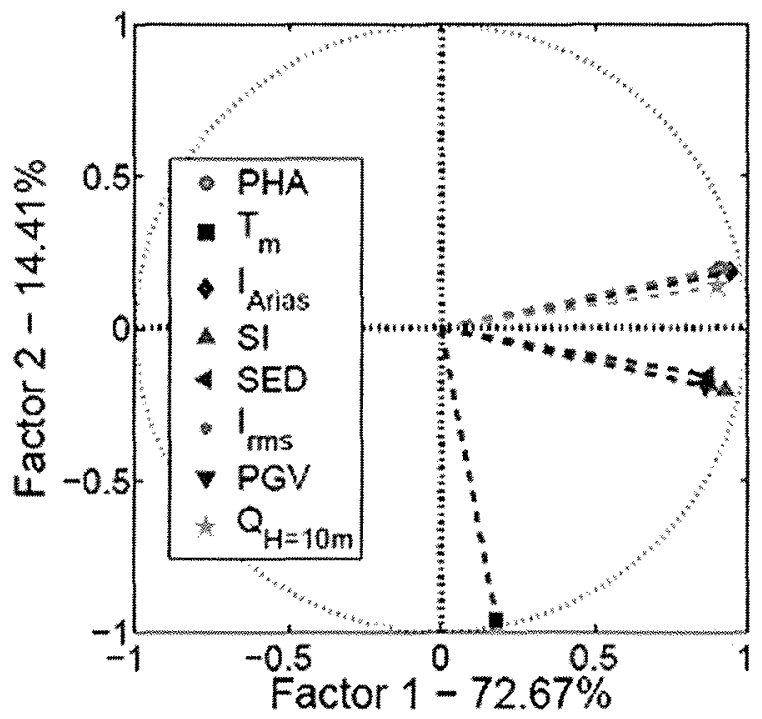

(a)
La variation de la valeur de $Q_{\mathrm{H}}=10 \mathrm{~m}$ à la fin du signal sismique avec l'intensité d'Arias à l'affleurement rocheux $\left(\mathrm{I}_{\text {Arias }}\right.$ ) est donnée sur la figure 10. Comme l’on pouvait s'y attendre, la valeur de $Q_{H}=10 \mathrm{~m}$ augmente lorsque la valeur de $\mathrm{I}_{\text {Arias }}$ augmente.

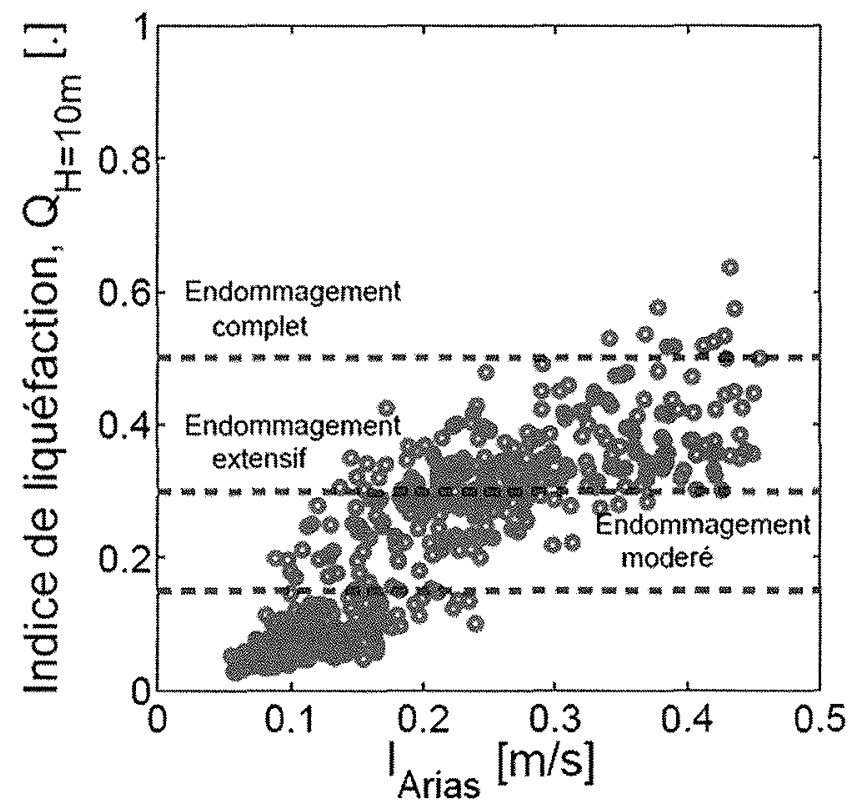

FG. 10 Diagramme de valeurs obtenues de $Q_{H}=10 \mathrm{~m}$ en fonction de $I_{\text {Arias }}$ (champ libre).

Dans le but d'étudier l'effet du séisme d'entrée sur l'amplitude de l'accélération obtenue à la surface, la variation de l'accélération maximale à la surface (PGA) avec l'accélération maximale à l'affleurement rocheux (PHA) est analysée (Fig. 11). D'après cette figure, une amplification de l'accélération à la surface par rapport au rocher apparaît pour des valeurs de PHA inférieures à 0,12 $\mathrm{g}$.

En ce qui concerne le contenu fréquentiel du signal à la surface, le spectre de réponse médian des signaux à la surface est donné sur la figure 12. Comme prévu, en raison de l'adoucissement du sol, pour des périodes plus grandes que $0,5 \mathrm{~s}$, ce dernier est supérieur à celui des signaux d'entrée.

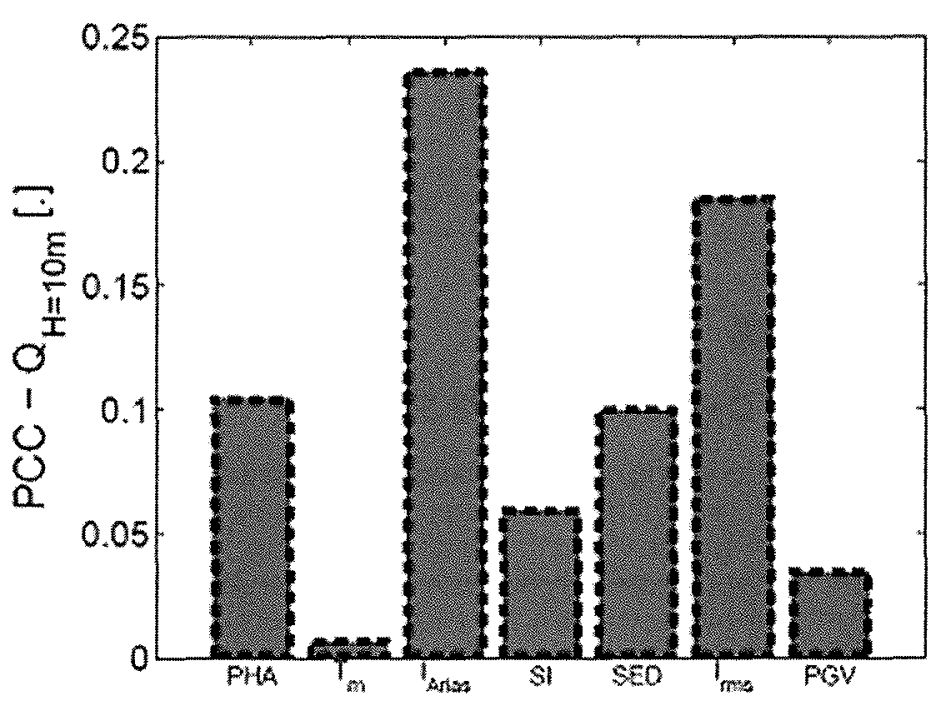

(b)

HG. 9 a) Analyse en composantes principales, plan factoriel et b) indices de corrélation partielle (PCC) expliquant $\mathrm{Q}_{\mathrm{H}}=10 \mathrm{~m}$. 


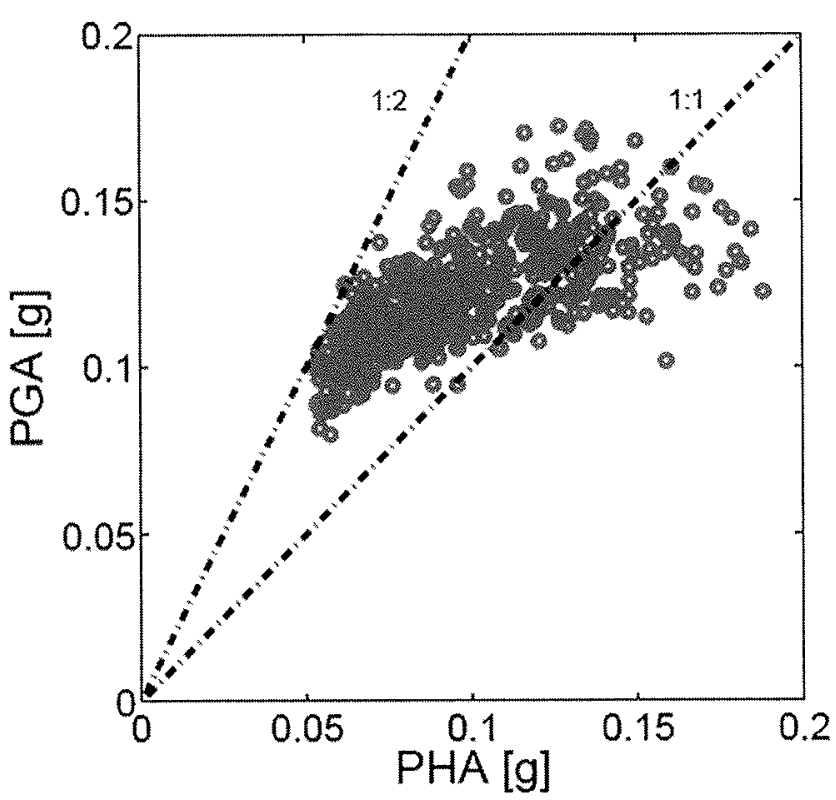

Fic 11 Relation entre l'accélération maximale à la surface (PGA) et celle au rocher affleurant (PHA) obtenue pour les séismes étudiés (champ libre).

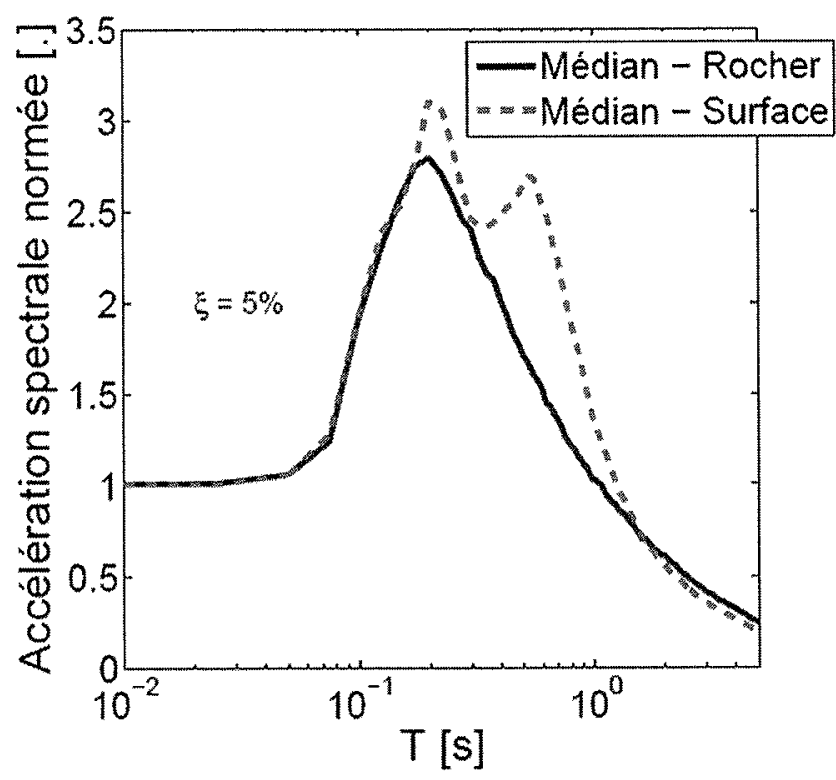

116. 12. Comparaison des spectres de réponse médians au rocher affleurant et en surface.

\section{3.}

\section{Réponse dynamique de la structure}

Pour cette partie, on utilise le modèle éléments finis complet (i.e. la structure, la fondation et le sol). A partir de chaque réponse dynamique de la structure, on peut obtenir le déplacement relatif maximal du sommet par rapport à la base pendant les calculs d'interaction sol structure (ISS). A partir des déplacements maximaux et des efforts tranchants correspondants transformés en valeurs spectrales (D-A), on peut tracer le spectre de capacité de la structure en dynamique, que l'on superpose à celle obtenue en statique (Fig. 13). On constate que les points obtenus sont approximativement sur la courbe de capacité. On observe aussi l'apparition d'un comportement non linéaire de la structure lors du chargement sismique (i.e. $\mathrm{D}>\mathrm{Dy}$, Dy étant le déplacement correspondant au seuil d'élasticité de la structure). Si l'on définit $\mu$ comme la ductilité $(\mu=\mathrm{D} / \mathrm{Dy}$ avec Dy $=0,51 \mathrm{~cm}$ ), on voit qu'elle varie entre 1,8 et 4,8 .

Concernant les tassements co-sismiques relatifs à celui du champ libre induits par la liquéfaction dans la structure, on peut noter que l'on obtient une valeur maximale de $10 \mathrm{~cm}$ (Fig. 14). On observe aussi que plus la valeur de l'intensité d'Arias à l'affleurement rocheux $\left(I_{\text {Arias }}\right.$ ) est élevée, plus le tassement relatif obtenu est grand. Selon Yasuda (2007), le tassement induit par la liquéfaction est produit principalement par le mouvement horizontal du sol sous la structure.

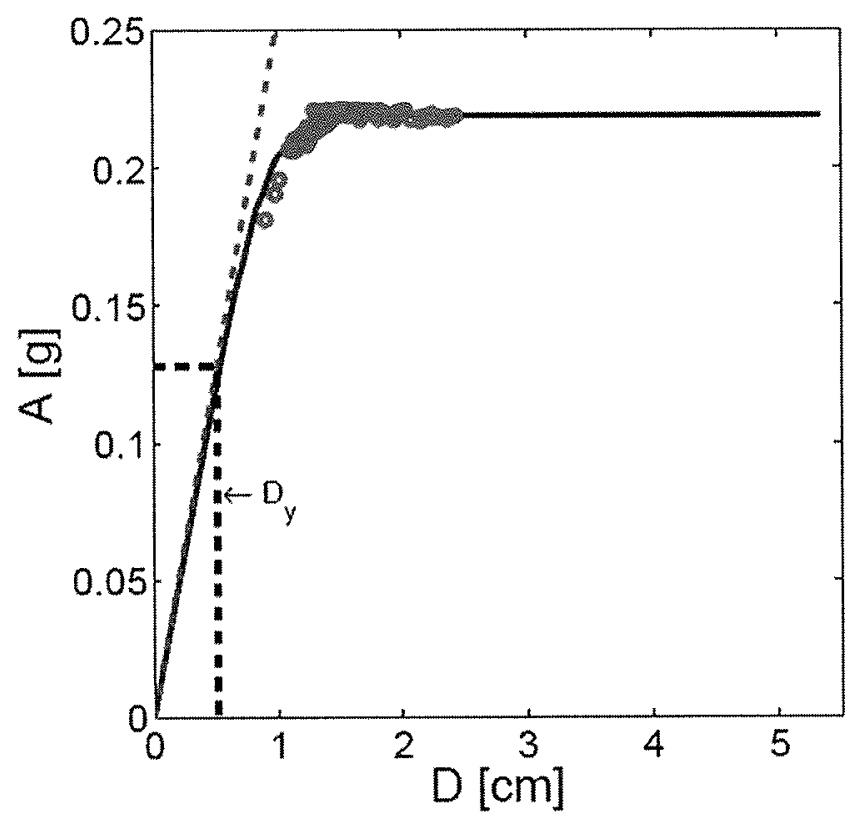

76. 13 Comparaison des spectres de capacité de la structure en dynamique (condition ISS) et en statique.

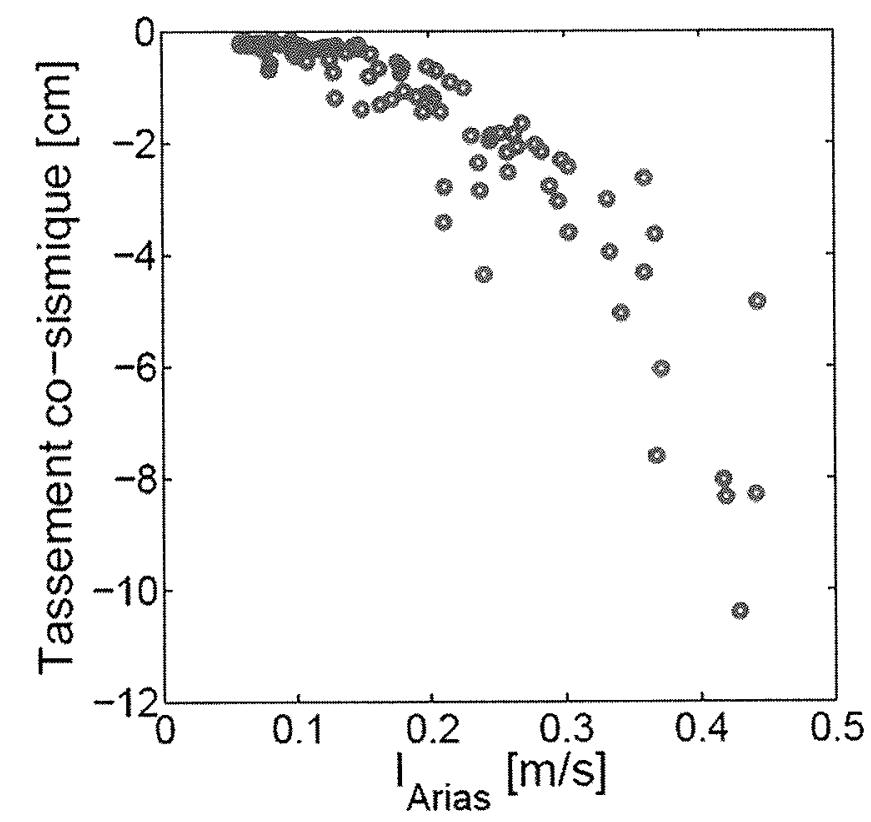

F6. 14 Diagramme de valeurs de tassement relatif co-sismique obtenues en fonction de $I_{\text {Arias }}$ (ISS). 


\section{Analyse de la méthode de réduction de la liquéfaction}

Dans cette section, une méthode pour la réduction du potentiel de liquéfaction (i.e. inclusions rigides) est étudiée afin d'améliorer le comportement du sol de fondation sous la structure. Cette méthode réduit le potentiel de liquéfaction en rigidifiant le sol latéralement et diminue le tassement en empêchant le mouvement horizontal du sol (i.e. en confinant la masse du sol sous la fondation). En ce qui concerne les murs de confinement, ils sont composés de deux inclusions avec une profondeur de $8 \mathrm{~m}$ et une épaisseur de $0,6 \mathrm{~m}$. La distance entre elles est de $6 \mathrm{~m}$ et il est supposé qu'elles sont fixées latéralement à la fondation.

Du point de vue de la modélisation, les inclusions sont simulées par des éléments poutre avec un modèle de comportement élastique isotrope et un module de Young, $E_{\text {inc }}=25,5 \mathrm{GPa}$ (Fig. 15). Elles sont supposées être étanches. L'interface entre le sol liquéfiable et le mur de confinement a été modélisée à l'aide des éléments d'interface avec « épaisseur zéro » et un modèle de comportement avec un critère de type Mohr-Coulomb non associé rigide parfaitement plastique. L'angle de frottement de l'interface est supposé être de $23^{\circ}$.

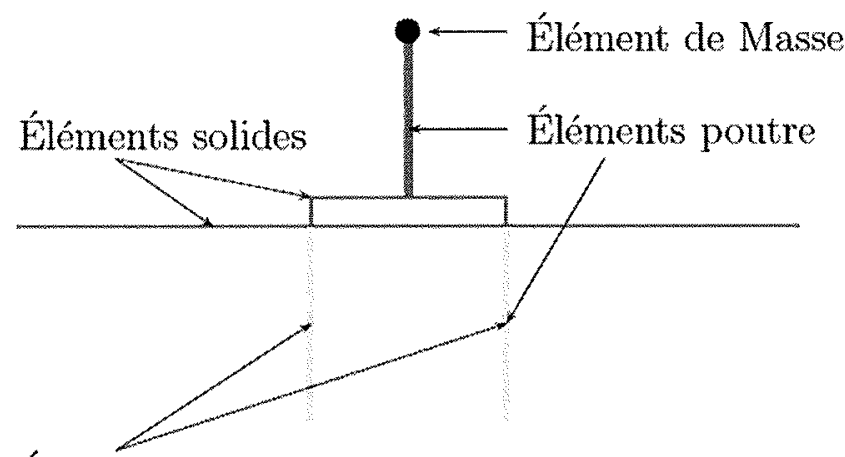

Éléments d'interface

fic is Schéma de la méthode d'amélioration de sols utilisée.

La distribution du rapport de surpression interstitielle ( $\mathrm{ru}=\Delta \mathrm{p}_{\mathrm{w}} / \sigma_{\mathrm{\gamma o}}^{\prime}$ ) en dessous de la fondation à la fin du mouvement sismique d'un cas avec et sans inclusions est présenté sur la figure 16. Une comparaison des distributions de ru des deux profils indique que, sous la fondation le niveau de ru diminue fortement lorsque les inclusions sont utilisées. Cependant, en dehors de la zone des inclusions, c'est-à-dire le champ libre, les valeurs de ru sont proches de 1,0 dans les deux cas et donc il semble qu'il n'y a pas d'effet.

Maintenant, sur la figure 17, on compare la valeur de la déformation de cisaillement maximale $\left(\gamma_{\max }\right)$ obtenue dans le profil de sol sous la fondation avant et après l'amélioration pour le même signal d'entrée. Il est intéressant de remarquer que dans la zone d'interaction des inclusions (i.e. $8 \mathrm{~m}$ ), les valeurs de $\gamma_{\max }$ diminuent lorsque les inclusions sont utilisées. Cela confirme que l'efficacité de cette méthode est due à l'effet de confinement du sol en limitant son écoulement latéral.
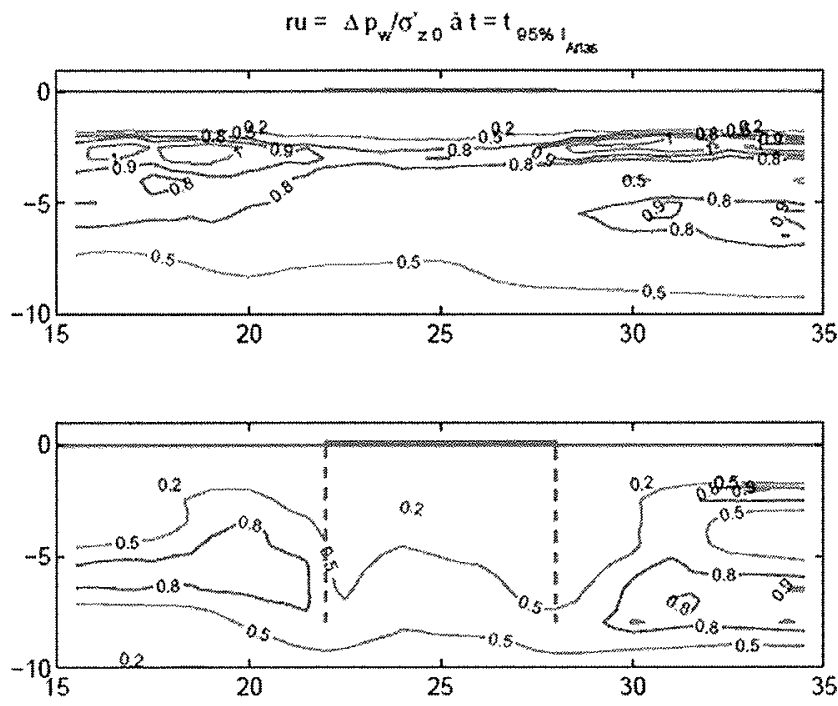

FI. 16. Comparaison de distribution du rapport de pression interstitielle sous la fondation avant et après l'amélioration.

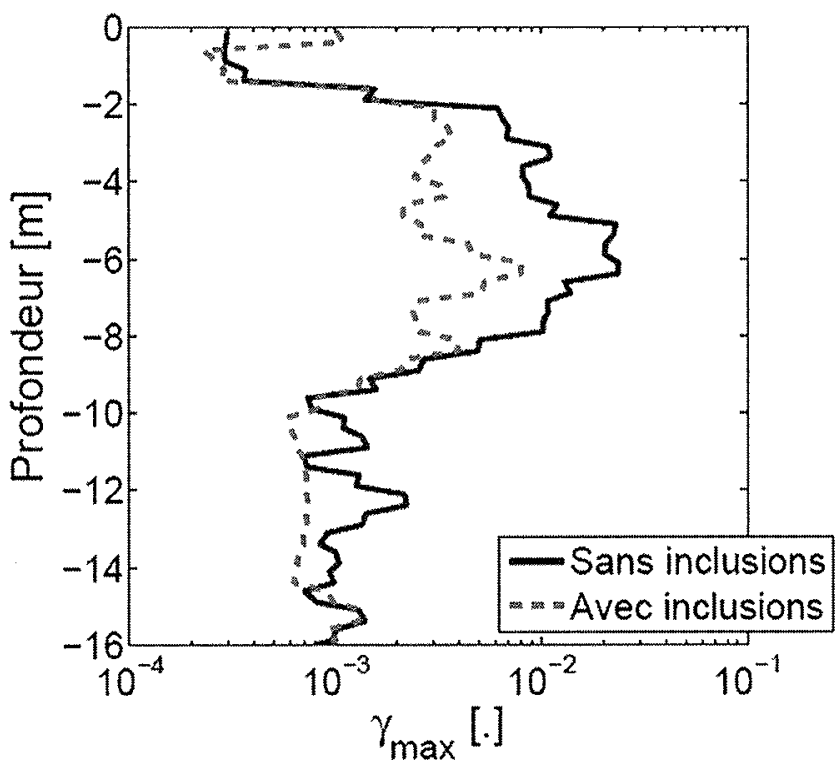

HG. 17 Déformation de cisaillement maximale $\gamma_{\max }$ obtenue dans le profil de sol sous la fondation avant et après l'amélioration.

Comme cela a été déjà mentionné, la méthode utilisée augmente la résistance à la liquéfaction et diminue le tassement de la structure. Comme l'illustre la figure 18 , les tassements relatifs co-sismiques de la structure obtenus après l'amélioration du sol sont fortement réduits grâce au confinement du sol.

Les effets bénéfiques ou défavorables de la méthode d'amélioration de sols sur le comportement de la structure peuvent être illustrés en comparant les réponses obtenues dans le profil avec et sans inclusions en utilisant des rapports tels que : $\Delta \mathrm{Q}=(\mathrm{Q}$ mit - Qo)/Qo pour le potentiel de liquéfaction et $\Delta \mu=(\mu \mathrm{mit}$ $-\mu o) / \mu o$ pour le comportement de la structure. Avec les indices ( mit ) et $(\mathrm{O})$ ) correspondant aux valeurs après et avant l'amélioration, respectivement. 


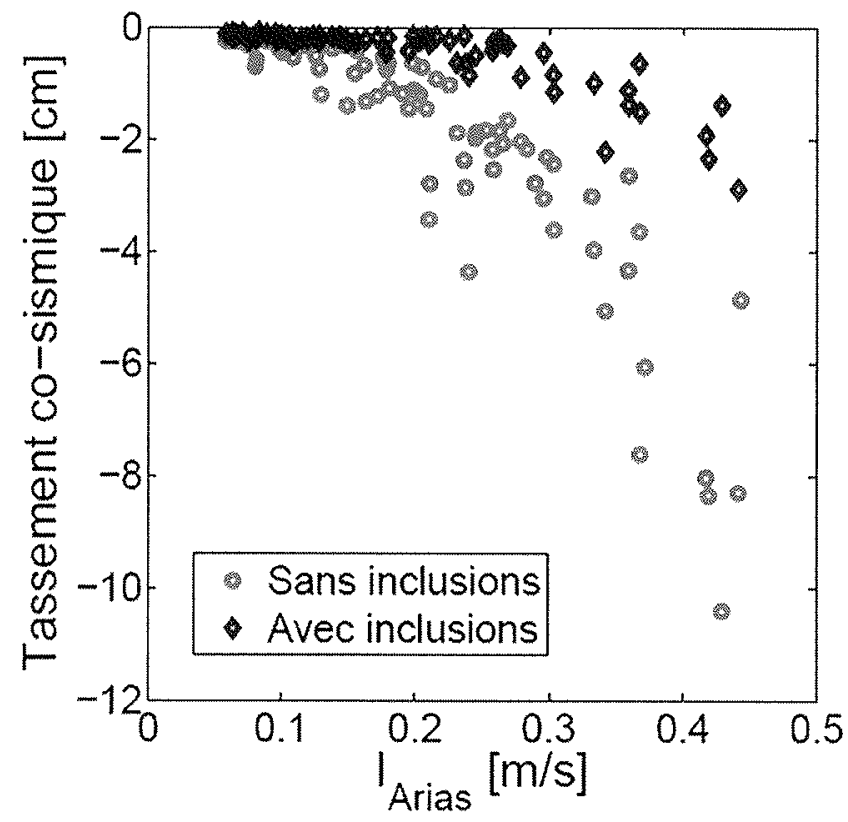

FIG. 18 Comparaison de valeurs obtenues de tassement co-sismique en fonction de $\mathrm{I}_{\text {Arias }}$ sans et avec inclusions.

Afin d'évaluer l'effet de l'utilisation des inclusions sur le comportement de la structure, on utilise le diagramme de la figure 19. On constate que, l'augmentation de la pression interstitielle et en conséquence le tassement, est réduite par la présence des inclusions (i.e. $\Delta \mathrm{Q} \leq 0$, zones II et III). Ce qui montre l'efficacité de la méthode concernant la liquéfaction. Cependant, concernant la variation de la ductilité $\mu$ de la structure, il apparaît que dans certains cas, elle augmente en raison de l'effet de (" rigidification ) du sol (i.e. $\Delta u \geq 0$, zones I et II), elle est par conséquent une méthode défavorable du point de vue structurel. Finalement, à en juger de ces résultats, en plus de l'amélioration du sol, il est également nécessaire de renforcer la structure afin d'empêcher des dégâts supplémentaires.

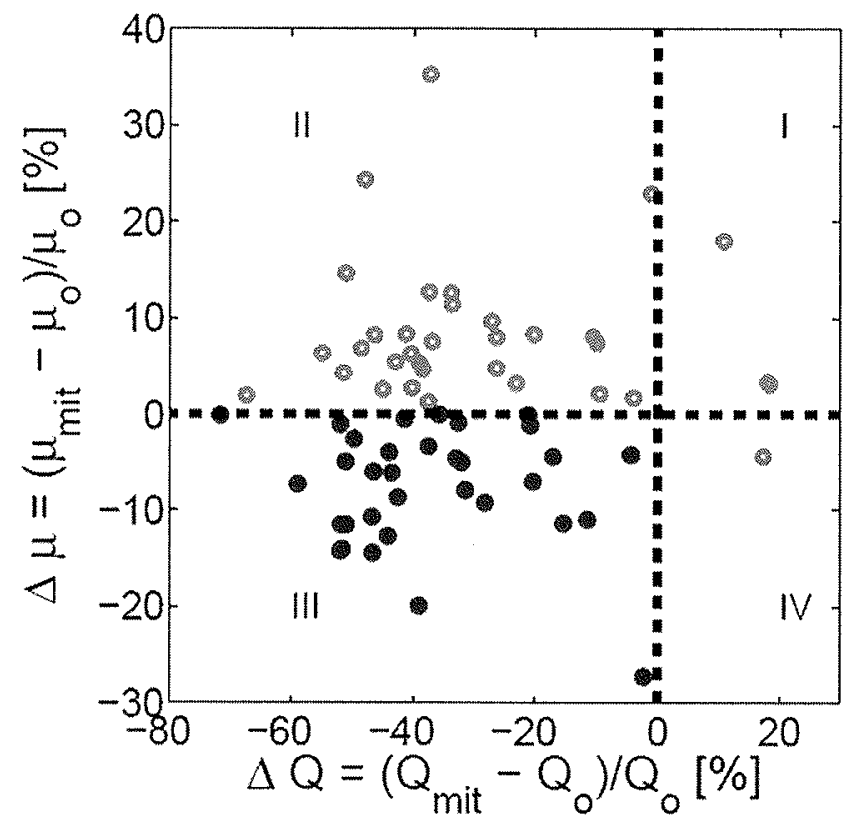

FG. 19 Diagramme de valeurs de la variation des rapports de ductilité $\Delta \mu$ par rapport à la variation du rapport de l'indice de liquéfaction $\Delta \mathbf{Q}$.
5

\section{Conclusion}

Une série d'analyses par éléments finis a été réalisée afin d'étudier les effets des mesures de réduction de la liquéfaction sur le comportement d'un profil de sol. Les principales conclusions tirées de cette étude sont les suivantes.

D’après les réponses obtenues avec le modèle sans inclusions, on peut conclure que le choix des signaux au niveau du rocher reste le paramètre le plus important pour définir les zones liquéfiables et les caractéristiques des contre-mesures possibles. Ainsi, une analyse paramétrique est nécessaire afin d'étudier l'influence de plusieurs paramètres du signal sur la réponse du profil de sol.

Les analyses ont montré que l'utilisation des inclusions rigides réduit la génération de pression interstitielle dans le profil du sol. En conséquence, pour un aléa sismique donné le tassement relatif de la structure diminue lorsque la méthode d'amélioration est utilisée. En revanche, elle augmente l'amplitude de la ductilité induite sur la structure ce qui pourrait être un désavantage d'un point de vue structurel.

\section{REMERCIEMENTS}

Cette étude a été réalisée dans le cadre du contrat ANR (BellePaline ANR-06-CATT-003) et du contrat de la Communauté Européenne No GRDI 40457-NEMISREF (New methods of mitigation of seismic risk on existing foundations).

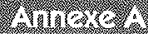

\section{Définition des caractéristiques des séismes d'entrée}

Les caractéristiques des séismes d'entrée sont obtenues comme suit, où a(t) est l'accélération en fonction du temps $(t), v(t)$ la vitesse en fonction de $t$, PSV le spectre de pseudo-vitesse, fi et Ci sont la i-ème fréquence et son amplitude correspondante de la transformé de Fourier et $t_{\text {IArias }}{ }^{n}$ le temps pour atteindre le $n \%$ de la valeur maximale d'I $I_{\text {Arias }}$ :

- Intensité d'Arias (I $\mathrm{I}_{\text {Arias }}$ ) (Arias, 1970),

$$
I_{\text {Arics }}=\frac{\pi}{2 \mathrm{~g}} \int_{0}^{\tau} a(t)^{2} d t
$$

- Période moyenne (Tm) (Rathje et al., 1998),

$$
T_{m}=\frac{\sum_{i}^{n} C_{i}^{2}\left(\frac{1}{f_{i}}\right)}{\sum_{i}^{n} C_{i}^{2}}
$$

- L'intensité spectrale (SI) (Kramer, 1996),

$$
S I(\xi)=\int_{0.1 s}^{2.5 s} P S V(\xi, T) d T
$$

- L'intensité rms ( $\mathrm{I}_{\mathrm{rms}}$ ) (Koutsourelakis et al., 2002),

$$
I_{r m s}=\sqrt{\frac{1}{\tau} \int_{0}^{r} a(t)^{2} d t}
$$

- La durée significative $\left(\mathrm{t}_{595}\right)$

$$
t_{595}=t_{I_{\text {Arias }}^{95}}^{95}-t_{I_{\text {Arias }}^{5}}
$$

- La densité d'énergie spécifique (SED),

$$
S E D=\int_{0}^{\tau} v(t)^{2} d t
$$




\section{Bibliographie}

Arias A. - A mesure of earthquake intensity. Seismic Design 297 for Nuclear Power Plants, R.J. Hansen (ed.), MIT Press, Cambridge, Massachusetts, 1970, p. $438-483$.

Aubry D., Chouvet D., Modaressi A., Modaressi H. - GEFDyn, Logiciel d'analyse de comportement mécanique des sols par éléments finis avec prise en compte du couplage sol-eau-air. Manuel scientifique, Ecole centrale Paris, LMSSMat., 1986

Aubry D., Hujeux J.-C., Lassoudière F., Meimon Y. - A double memory model with multiple mechanisms for cyclic soil behaviour. Int. Symp. Num. Mod. Geomech, Balkema, 1982, p. 3-13.

Aubry D., Modaressi A. - GEFDyn. Manuel scientificue, École centrale Paris, LMSSMat., 1996

Bird J.F., Bommer J.J., Crowley H., Pinho R. - Modelling liquefactioninduced building damage in earthquake loss estimation. Soil Dynamics and Earthquake Engineering, 26 (1), 2006 , p. 15-30.

Hujeux J.-C. - Une loi de comportement pour le chargement cyclique des sols. Génie parasismique (ss la dir. de V. Davidovici), Presses des Ponts, 1985, p. 278302

Juang C.H., Yuan H., Li D.K., Yang S.H. Christopher R.A. - Estimating severity of liquefaction-induced damage near foundation. Soil Dynamics and Earthquake Engineering, 25 (5), 2005, p. 403411.

Katona M.G., Zienkiewicz O.C. - A unified set of single step algorithms. Part 3 : The beta-m method, a generalization of the newmark scheme. International Journal of Numerical Methods in Engineering, 21 (7), 1985, p. 1345-1359

Koutsourelakis S., Prévost J.H., Deodatis G. - Risk assessment of an interacting structure-soil system due to liquefaction. Earthquake Engineering and Structural Dynamics, 31 (4), 2002, p. 851-879.

Kramer S.L. - Geotechnical Earthquake Engineering. Prentice Hall, Upper Saddle River, NJ, 1996.
Lopez-Caballero F., Modaressi-Farahmand-Razavi A. - Numerical simulation of licuefaction effects on seismic SSI. Soil Dynamics and Earthquake Engineering, 28 (2), 2008, p. 85-98.

Lopez-Caballero F., Modaressi-Farahmand-Razavi A., Modaressi H. - Nonlinear numerical method for earthquake site response analysis I- elastoplastic cyclic model \& parameter identification strategy. Bulletin of Earthquake Engineering, 5 (3), 2007, p. 303-323.

Martin P.P., Seed H.B. - Simplified procedure for effective stress analysis of ground response. Journal of the Geotechnical Engineering Division ASCE, 105 (GT6), 1979, p. 739-758.

Modaressi H., Benzenati I. - Paraxial approximation for poroelastic media. Soil Dynamics and Earthquake Engineering, 13 (2), 1994, p. 117-129.

Modaressi-Farahmand-Razavi A. - Modélisation numérique : un outil efficace pour analyser le comportement des ouvrages. Du sol à l'ouvrage : une vision multiéchelles de la géomécanique (ss la dir. de P.-Y. Hicher et E. Flavigny), Traité MIM Série géomatériaux, Hermès-Lavoisier, 2010, p. 277-370.

NRC - Liquefaction of soils during earthquakes. Report No. CETS-EE-001, Comittee on Earthquake Engineering, National Research Council, Washington, DC, 1985

Pousse G., Bonilla F., Cotton F., Marcjerin L. Non stationary stochastic simulation of strong ground motion time histories including natural variability : Application to the K-net Japanese database. Bulletin of the Seismological Society of America, 96 (6), 2006, p. 2103-2117

Rathje E.M., Abrahamson N.A., Bray J.D. Simplified frequency content estimates of earthquake ground motions. Journal of Geotechnical and Geoenvironmental Engineering, 124 (2), 1998, p. 150-159.

Saez. E. - Dynamic non-linear Soil-Structure Interaction. PhD thesis, École centrale Paris, France, 2009

Santos J.A., Gomes Correia A., Modaressi A., Lopez-Caballero F., Car- rilho Gomes R. - Validation of an elas toplastic model to predict secant shear modulus of natural soils by experimental results. Deformation Characteristics of Geomaterials. H. Di Benedetto et al (Eds), A.A. Balkema, 2003, p. 1057-1061.

Seed H.B., Idriss I.M. - Simplified procedure for evaluating soil liquefaction potential. Journal of Soil Mechanics and Foundations Division ASCE, 97 (SM9), 1971, p. 1249-1273.

Seed H.B., Idriss I.M. - Ground motion and soil liquefaction during earthquakes. Monograph series, earthquake engineering research institute, University of California, Berkeley, CA, 1982.

Seed H.B., Idriss I.M., Arango I. - Evaluation of liquefaction potential using field performance data. Journal of Geotechnical Engineering, ASCE, 109 (3), 1983, p. 458-482.

Seed H.B., Wong R.T., Idriss I.M., Tokimatsu K. - Moduli and damping factors for dynamic analyses of cohesionless soils. Journal of Geotechnical Engineering, ASCE, 112 (11), 1986, p. 1016-1032.

Shinozuka M. Ohtomo K. - Spatial severity of liquefaction. Proceedings of the second US-Japan workshop in liquefaction, Large Ground Deformation and Their Effects on Lifelines, 1989.

Yasuda S. - Remediation methods againts liquefaction which can be applied to existing structures. Earthquake Geotechnical Engineering, K.D. Pitilakis Ed., Springer, The Netherlands, 2007, p. 385 406.

Youd T.L., Idriss I.M. Andrus R.D., Arango I., Castro G., Christian J.T., Dobry R., Finn W.D.L., Harder L.F.J. Hynes M.E., Ishihar K., Koester J.P. Liao S.S.C., Marcuson W.F I., Martin G. Mitchell J.K., Moriwaki Y., Power M.S., Robertson P.K., Seed R.B., Stokoe K.H.I - Liquefaction resistance of soils: Sum mary report from the 1996 NCEER and 1998 NCEER/NSF workshops on evalua tion of liquefaction resistance of soils Journal of Geotechnical and Geoenvironmental Engineering - ASCE, 127 (10), 2001, p. 817-833. 